Research Paper

\title{
Tumoral Pyruvate Kinase L/R as a Predictive Marker for the Treatment of Renal Cancer Patients with Sunitinib and Sorafenib
}

\author{
Marjut Niinivirta1,2, Gunilla Enblad1,2, Cecilia Lindskog33, Fredrik Pontén3, Anca Dragomir ${ }^{3,4}$ and Gustav J. \\ Ullenhag $1,2 \bowtie$ \\ 1. Department of Immunology, Genetics and Pathology, Uppsala University, Uppsala, Sweden \\ 2. Department of Oncology, Uppsala University Hospital, Entrance 78, 75185 Uppsala, Sweden \\ 3. Department of Immunology, Genetics and Pathology and Science for Life Laboratory, Uppsala University, Dag Hammarskjölds väg 20, 751 85 Uppsala, \\ Sweden. \\ 4. Department of Surgical Pathology, Uppsala University Hospital, 75185 Uppsala, Sweden. \\ $\bowtie$ Corresponding author: Gustav J. Ullenhag, MD, Associate Professor, Tel: +46 18611 00 00; Fax: +46 1861155 28; E-mail: Gustav.Ullenhag@igp.uu.se \\ (c) Ivyspring International Publisher. This is an open access article distributed under the terms of the Creative Commons Attribution (CC BY-NC) license \\ (https://creativecommons.org/licenses/by-nc/4.0/). See http://ivyspring.com/terms for full terms and conditions.
}

Received: 2018.09.22; Accepted: 2019.04.26; Published: 2019.06.02

\begin{abstract}
Background and aims: Treatment with tyrosine kinase inhibitors (TKI) like sunitinib and sorafenib has improved the prognosis of patients with metastatic renal cell cancer (mRCC). No predictive marker is available to select patients who will gain from these treatments. Tumoral pyruvate kinase $L / R$ (PKLR) is a membrane protein with highly specific expression in the renal tubule. We have previously shown that the tumoral expression of cubilin (CUBN) is associated with progression free survival (PFS) in mRCC patients treated with sunitinib and sorafenib. The aim of the present study was to investigate if PKLR can predict response in these patients, alone and/or in combination with CUBN.

Methods: A tissue microarray (TMA) was constructed of tumor samples from $139 \mathrm{mRCC}$ patients. One hundred and thirty-six of these patients had been treated with sunitinib or sorafenib in the first or second-line setting. Thirty patients suffered from early severe toxicity leading to the termination of treatment. The remaining patients $(n=106)$ were selected for the current study.

Results: Fifty-five (52\%) of the tumors expressed membranous PKLR. Patients with PKLR tumor expression experienced a significantly longer PFS compared to patients with no expression (eight versus five months, $p=0.019$ ). Overall survival $(O S)$ was also significantly better for patients with PKLR expression. In addition, the combined expression of PKLR and CUBN resulted in a higher predictive value than either marker alone.

Conclusions: In this real world study we show that tumoral PKLR membrane expression is a positive predictive biomarker for sunitinib and sorafenib treatment in patients suffering from $\mathrm{mRCC}$. Our results also indicate that the combined expression with cubilin more accurately than PKLR alone can select patients with no benefit from treatment.
\end{abstract}

Key words: PKLR, predictive marker, renal cancer, tyrosine kinase inhibitor

\section{Introduction}

Since chemo- and radiotherapy has not been a successful way to treat patients with metastatic renal cell cancer (mRCC) (1) and cytokine treatment has shown an objective response rate of $7,5 \%$ only, the prognosis for these patients has been very poor (2).
However, tyrosine kinase inhibitors (TKIs) have increased the treatment arsenal. Sunitinib is recommended for treatment naïve mRCC patients and increases the median progression free survival (PFS) from 5 to 11 months in the first line setting $(3,4)$. 
Another common used TKI, sorafenib, extends the median PFS in the second line setting to 5.5 months compared with 2.8 months for placebo treated patients. Sunitinib and sorafenib inhibit vascular endothelial growth factor (VEGF) receptors as well as platelet-derived growth factor (PDGF) receptors found intracellularly (5).

Though the introduction of TKI treatment has improved the prognosis for these patients there are still some dilemmas to solve. TKIs can cause a variety of more or less serious side effects and all the patients do not gain from the treatment (6). The price of the medication is also high. To avoid treating mRCC patients with no benefit of TKIs, a predictive marker is needed.

Heng's prognostic factors (Karnofsky performance status, time from primary diagnosis to treatment start, hemoglobin, neutrophils and platelet counts and serum calcium) are known risk factors to estimate the survival irrespective of treatment (7). Some clinical side effects of TKIs (hypertension and hand-foot skin reaction) are associated with better outcome in mRCC patients. These side effects provide an opportunity to early assess whether the patient benefits. However, a pretreatment biomarker being able to accurately select patients for the treatment would be much more valuable $(8,9)$.

Hence, what is urgently needed is a predictive factor to guide us in the clinical practice. There is no established predictor for TKI treatment in $\mathrm{mRCC}$ patients.

The majority of predictive marker studies in mRCC patients have been conducted in sunitinib treated patients and have mainly investigated the predictive value of protein levels in serum. For tumor necrosis factor a (TNFa), metalloproteinase-9 (MMP-9), circulating VEGF and neutrophil gelatinase associated lipocalin (NGAL), associations to sunitinib response have been demonstrated in small studies (10, 11). Plasma levels of VEGF and circulating cell-free DNA (cfDNA) were measured in other small studies in trying to predict response to sorafenib-treatment $(12,13)$. However, we wanted to investigate the actual drug target, the tumor tissue, since the levels of potential biomarkers in the blood are dependent of other factors than the tumor. In a tissue microarray (TMA), hundreds of tumor cores are sampled together and can be analyzed at the same time.

Pyruvate kinase (PK) is an enzyme that catalyzes phosphoenolpyruvate (PEP) to adenosine diphosphate (ADP) in glycolysis which results in one pyruvate molecule and one adenosine triphosphate (ATP) (14). Transcription of the PK gene is regulated in an opposing manner by nuclear factor (NF) 1 family members and hepatocyte nuclear factor (HNF) 4 (15).
One of the four isoenzymes of PK, pyruvate kinase muscle 2 (PKM2) regulates glucose metabolism due to the needs of the cell under changing conditions (16). Other studies indicate that cancer cells involve both PKM1 and PKM2 to drive the glycolysis (17).

The Warburg effect is observed in cancer cells, favoring glycolysis over mitochondrial oxidative phosphorylation, even in the presence of oxygen (i.e. aerobic glycolysis). The energy production is less efficient by this phenomenon but it generates metabolites needed for rapid tumor proliferation such as nucleotides and amino acids. Mineralocorticoid receptor (MR) is present in a wide variety of tissues, having many different functions. In hepatocellular cancer, MR was shown to regulate transcription of microRNA (miRNA)-338-3p which directly targets another isoenzyme of PK, pyruvate kinase liver and red blood cells (PKLR), thereby suppressing the Warburg effect and cancer progression $(18,19)$.

Over 250 PKLR-gene mutations have been described and Alu retrotransposons are the most abundant and have been associated with a number of human diseases (20).

Studying colorectal cancer, PKLR negatively regulated PKM2 which in addition to its role in glycolysis balances the synthesis of the major endogenous antioxidant, glutathione. Pyruvate kinase liver and red blood cells increased glutathione and advanced tumor cell survival (21).

Expression of the PK-regulating enzyme pyruvate dehydrogenase kinase-1 (PDK-1) was investigated in tumor and corresponding normal tissue from patients with clear cell RCC. Rather conflicting results were observed: PDK-1 was up-regulated in RCC cells compared to normal renal tissue. However, PDK-1 expression was lower with increasing tumor stage. An association between PDK-1 expression and PFS and OS was also observed, even after adjustment for stage and grade, suggesting a prognostic role of PDK-1 in RCC (22).

In RCC patients, circulating levels of PKM2 were significantly associated with both tumor stage and tumor grade. In this study, elevated levels of PKM before nephrectomy were suggested as a negative predictor for recurrence (23).

Through The Human Protein Atlas (http://www.proteinatlas.org) internal database for proteins PKLR was discovered as a potentially interesting biomarker. Pyruvate kinase liver and red blood cells were selected for this study based on the highly specific expression in renal tubule of normal kidney and in RCC. Expression of PKLR is also noticed in hepatocytes and hematopoietic cells (24).

The aim of the present study was to investigate the potential value of tumoral expression of PKLR as a 
predictor for TKI treatment in mRCC patients. We also examined the predictive role of the combination of membranous PKLR and cubilin (CUBN). We have recently demonstrated that CUBN is a prognostic and predictive factor in this patient group $(25,26)$.

\section{Material and Methods}

\section{Patients}

The local Research Ethics Committee granted approval for the study (2009/139). Patients still alive gave their written informed consent. The TMA cohort has been described previously $(26,27)$ consisting of 139 mRCC patients diagnosed with mRCC between 2006 and 2010. The patients were localized from seven Departments of Oncology in Sweden: Uppsala $(n=48)$, Göteborg $(n=36)$, Örebro $(n=19)$, Västerås $(n=12)$, Gävle $(n=11)$, Falun $(n=7)$ and Karlstad $(n=6)$. All the patients had a primary surgery (nephrectomy) at diagnosis and received various systemic therapeutic agents in the metastatic stage of the disease.

The patients $(n=136)$ treated with sunitinib or sorafenib in the first or second line setting were selected for the current study. Twenty of these received IFN- $\alpha$ as initial systemic treatment.

Clinical data were captured, including the patient's age, gender and histologic subtype (Table 1) (26) as well as the length of treatment with sunitinib and sorafenib from hospital medical records. Progression free survival (defined clinically and/or radiologically) was selected as the primary endpoint and was calculated as the time from the start of treatment to the time of clinical and/or radiological progression, treatment discontinuation due to toxicity, death or end of follow up. Overall survival was selected as the secondary endpoint.

\section{Tissue microarray generation, immunohistochemical methods and slide scanning}

Tissue microarray (TMA), immunohistochemistry and slide scanning were essentially performed in accordance to standards used in the Human Protein Atlas (www.proteinatlas.org) at the Swedish Science for Life Laboratory (SciLifeLab) facilities in the Department of Immunology, Genetics, and Pathology at the Rudbeck Laboratory of Uppsala University and has been previously described $(24,26$, 28). In brief, corresponding HE slides were examined and representative tumor parts chosen for the TMA. For each patient, two $1 \mathrm{~mm}$ diameter cores containing tumor tissue were collected by punch biopsy and transferred to recipient paraffin blocks subsequently containing 277 cores. TMArrayer ${ }^{\mathrm{TM}}$ (Pathology Devices, Westminster, MD, USA) and the Beecher
Instruments Manual Tissue Arrayer MTA-1 (Estigen OÜ, Tartu, Estonia) were used for this procedure.

Table 1. Clinical characteristics of renal cancer patients treated for metastatic disease with sunitinib or sorafenib in the first or second line setting (26)

\begin{tabular}{ll}
\hline Patient cohort & Total $\mathrm{n}=106$ \\
\hline Gender, $\mathrm{n}(\%)$ & $77(73)$ \\
Male & $29(27)$ \\
Female & \\
Age at diagnosis, years & $62,5(33-77)$ \\
Median (range) & \\
Age at metastatic disease, years & $65(34-84)$ \\
Median (range) & \\
Histologictype, $\mathrm{n}(\%)$ & $89(84)$ \\
Clear cell & $4(4)$ \\
Papillary & $4(4)$ \\
Mixed phenotype & $9(8)$ \\
Unknown & $49(46)$ \\
Local disease at diagnosis, $\mathrm{n}(\%)$ & $57(54)$ \\
Metastatic disease at diagnosis, $\mathrm{n}(\%)$ & \\
Time to metastasis, years & $2(0-18)$ \\
Median (range) & $20(41)$ \\
Metastasis during first year, $\mathrm{n}(\%)$ & $29(59)$ \\
Metastasis after first year, $\mathrm{n}(\%)$ & $17(16)$ \\
Alive, $\mathrm{n}(\%)$ & $89(84)$ \\
Dead, $\mathrm{n}(\%)$ & \\
\end{tabular}

TMA sections were deparaffinized in xylene, hydrated in graded alcohols and blocked for endogenous peroxidase in $0.3 \%$ hydrogen peroxide diluted in $95 \%$ ethanol. For antigen retrieval, a Decloaking chamber ${ }^{\circledR}$ (Biocare Medical, Walnut Creek, CA) was used. Slides were immersed and boiled in Citrate buffer ${ }^{\circledR}$, pH6 (Lab Vision, Freemont, CA) for $4 \mathrm{~min}$ at $125 \mathrm{C}$ and then allowed to cool down to $90 \mathrm{C}$ (the whole cycle is approximately 40 minutes). Automated immunohistochemistry was performed using an Autostainer 480 instrument ${ }^{\circledR}$ (Thermo Fischer Scientific, Waltham, Massachusetts, United States). The primary rabbit polyclonal antibody towards PKLR (HPA006653, Atlas Antibodies AB, Bromma, Sweden) was diluted in 1:1000 UltraAb Diluent (Thermo Fisher Scientific) followed by incubation for $30 \mathrm{~min}$ at room temperature (RT). The slides were further incubated with the secondary reagent anti-rabbit/mouse horse reddish peroxidase-conjugated UltraVision (Thermo Fischer Scientific) for $30 \mathrm{~min}$ at RT, and developed for $10 \mathrm{~min}$ using Diaminobenzidine (DAB) Quanto (Thermo Fisher Scientific) as chromogen. All incubations were followed by rinse in wash buffer ${ }^{\circledR}$ (Thermo Fisher Scientific) $2 \times 5$ min. Slides were counterstained in Mayers hematoxylin (Histolab) and cover slipped using Pertex ${ }^{\circledR}$ (Histolab) as mounting medium. High-resolution digital images were generated with an Aperio AT2 slide scanner (Aperio, Vista, CA), using a $20 x$ objective (26). 


\section{Slide scanning and evaluation of staining}

To obtain high-resolution digital images, the IHC slides were scanned with a 20x objective using the AperioScanScope XT Slide Scanner (Aperio Technologies, Vista, CA, USA).The digital images were examined in duplicates on a colour-calibrated screen using ImageScope (Aperio, Vista, CA, USA). Staining of the viable tumor cells was semi-quantitatively evaluated by two observers, of which one pathology specialist (MN and AD), blinded to the individual patient information, and disagreements were resolved by re-evaluation of the images. Two cellular compartments were annotated: cytoplasm and membrane. For the membrane staining, both extent (circumference) and fraction of stained cells were categorically estimated using a scale of $0-2$ for the extent $(0=$ negative $=0-10 \%$ of the cells circumference stained, $1=$ incomplete $=11-80 \%$ of the cells circumference stained, $2=$ complete $=81-100 \%$ of the cells circumference stained) and $0-4$ for the fraction of positive tumor cells $(0=0-1 \%, 1=2-25 \%$, $2=26-50 \%, 3=51-75 \%, 4=76-100 \%$ ).

The distribution of the results for the membranous expression is given in Table 2 .

Representative examples of negative, incomplete and complete membranous staining are shown in Figure 1.

\section{Statistical methods}

For statistical analysis, the combined immune score for a cellular compartment was calculated by addition of the extent score and fraction score, with a resulting scale from 0 to 6 for membrane. The median combined immune score for membranous staining was 3 and this value was selected as the cut-off for PKLR negative tumors (immune scores 0-2) versus PKLR positive tumors (immune scores 3-6).

Statistical analyses (Kaplan-Meier method, log-rank test) were performed using STATISTICA program (version 2016). A two sided p-value $<0.05$ was defined as statistically significant.
Table 2. Distribution of membrane staining results among primary tumours of renal cancer patients treated for metastatic disease with sunitinib or sorafenib in first or second line setting

\begin{tabular}{lll}
\hline \multicolumn{2}{l}{ Extent (circumference) } & of stained membrane and distribution \\
EXTENT SCORE & PERCENTAGE STAINED & NUMBER OF CASES \\
0 & $0-10 \%$ & 44 \\
1 & $11-80 \%$ & 6 \\
2 & $81-100 \%$ & 56
\end{tabular}

Fraction of stained membrane and distribution

FRACTION SCORE PERCENTAGE STAINED NUMBER OF CASES

$0-1 \%$

$1-2-25 \% \quad 16$

$2-26-50 \%-21$

$31-75 \% \quad 10$

Combined score (addition of extent and fraction score) and distribution COMBINED SCORE NUMBER OF CASES

$\begin{array}{ll}0 & 44 \\ 1 & 1 \\ 2 & 6 \\ 3 & 15 \\ 4 & 19 \\ 5 & 10 \\ 6 & 11\end{array}$

PKLR negative and positive cases

PKLR EXPRESSION NUMBER OF CASES

$(-) \quad 51$

$(+) \quad 55$

\section{Results}

\section{Patient population}

Treatment characteristics have previously been described (26) and are summarized in Table 3. Of all 139 patients in the TMA, 136 were treated with sunitinib or sorafenib in the first or second line setting. Thirty patients were excluded from analysis due to early side effects which lead to termination of therapy already before first treatment evaluation. Therefore, 106 patients were included in the final study cohort.

Sixty-one patients were treated with sunitinib and 45 with sorafenib in the final cohort. The median duration of TKI treatment was 7 months (range $0.5-40)$.

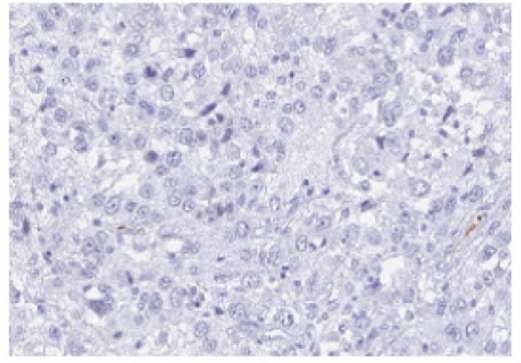

A

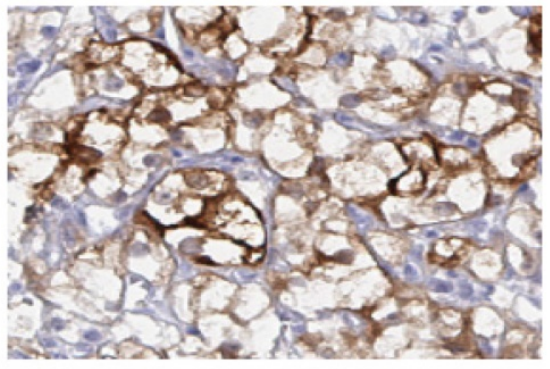

B

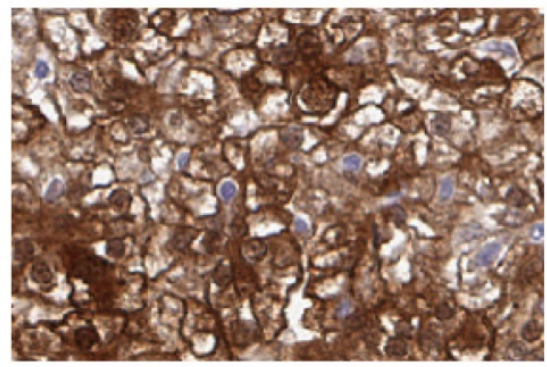

C

Figure 1. Representative immunohistochemical images of PKLR expression in primary renal cell carcinomas, from patients later treated for metastatic disease with sunitinib or sorafenib in the first or second line setting, demonstrating negative (A), incomplete (B) and complete (C) membranous staining in tumor cells. Magnification 200x 
Table 3. Treatment characteristics for renal cancer patients treated for metastatic disease with sunitinib or sorafenib in the first or second line setting (26)

\begin{tabular}{ll}
\hline Treatment & Total $\mathbf{n}=\mathbf{1 3 6}$ \\
\hline Sunitinib, $\mathrm{n}(\%)$ & $77(57)$ \\
First line setting & 66 \\
Second line setting & 11 \\
Sorafenib, $\mathrm{n}(\%)$ & $59(43)$ \\
First line setting & 45 \\
Second line setting & 14 \\
Side effects leading to discontinuation of & $30(22)$ \\
treatment, $\mathrm{n}(\%)$ & \\
Sunitinib & 16 \\
Sorafenib & 14 \\
Treated until progression/end of follow-up, $\mathrm{n}$ & $106(78)$ \\
(\%) & \\
Sunitinib & 61 \\
Sorafenib & 45 \\
Median PFS, months (range) & $7(0,5-40)$ \\
Sunitinib & 8 \\
Sorafenib & 6 \\
& \\
Still under treatment, $\mathrm{n}(\%)$ & $12(11)$ \\
\hline
\end{tabular}

Patient characteristics have been previously described (26) and are summarized in Table 1. The majority of patients were male $(n=77$, female $n=29)$ and were in median 65 years at the diagnosis of mRCC. Fifty-seven of 106 patients had a metastatic disease already at diagnosis.

The median OS was 26.5 months (range 1-144 months). Eighty-four percent $(n=89)$ had died at the end of the follow-up.

\section{PKLR expression}

No correlation with cytoplasmic PKLR staining was found (data not shown). For the membranous staining, using the median expression of PKLR as cut off, 55/106 (52\%) of the patients were PKLR positive.
A significant association with longer PFS was identified for patients with PKLR positive tumors $(\mathrm{p}=$ 0.019 , Figure 2). The median PFS was 8 months (range 1-40 months) for PKLR + patients and 5 months (range 0.5-34 months) for PKLR - patients.

No significant correlation between patient gender or age at diagnosis of $\mathrm{mRCC}$ and the membranous expression of PKLR was found ( $p$-values of 0.14 and 0.17 respectively).

The secondary end point, OS, was also significantly different between PKLR + and PKLR cases $(p=0.001$, Figure 3$)$. Almost twice as long median OS was measured in PKLR positive patients (31 months, range 4-144 months) compared to PKLR negative patients (17 months, range 1-84 months).

While combining expression of PKLR and CUBN, another known membrane protein in renal tubule, we found a higher predictive value than with PKLR alone. Patients with no expression of PKLR and CUBN in their primary tumors were treated for four months in median with TKIs compared with the other patients reaching a PFS of eight months $(p=0.007$, Figure 4).

\section{Discussion}

About $20 \%$ of RCC patients have already metastatic disease by diagnosis and one-third of patients with localized RCC develop distant spread later (4). Tyrosine kinase inhibitors like sunitinib and sorafenib are widely used to treat these patients to prolong PFS and OS but the treatment is associated with adverse events and some respond poorly to the medication $(5,29,30)$. Therefore, the need of a

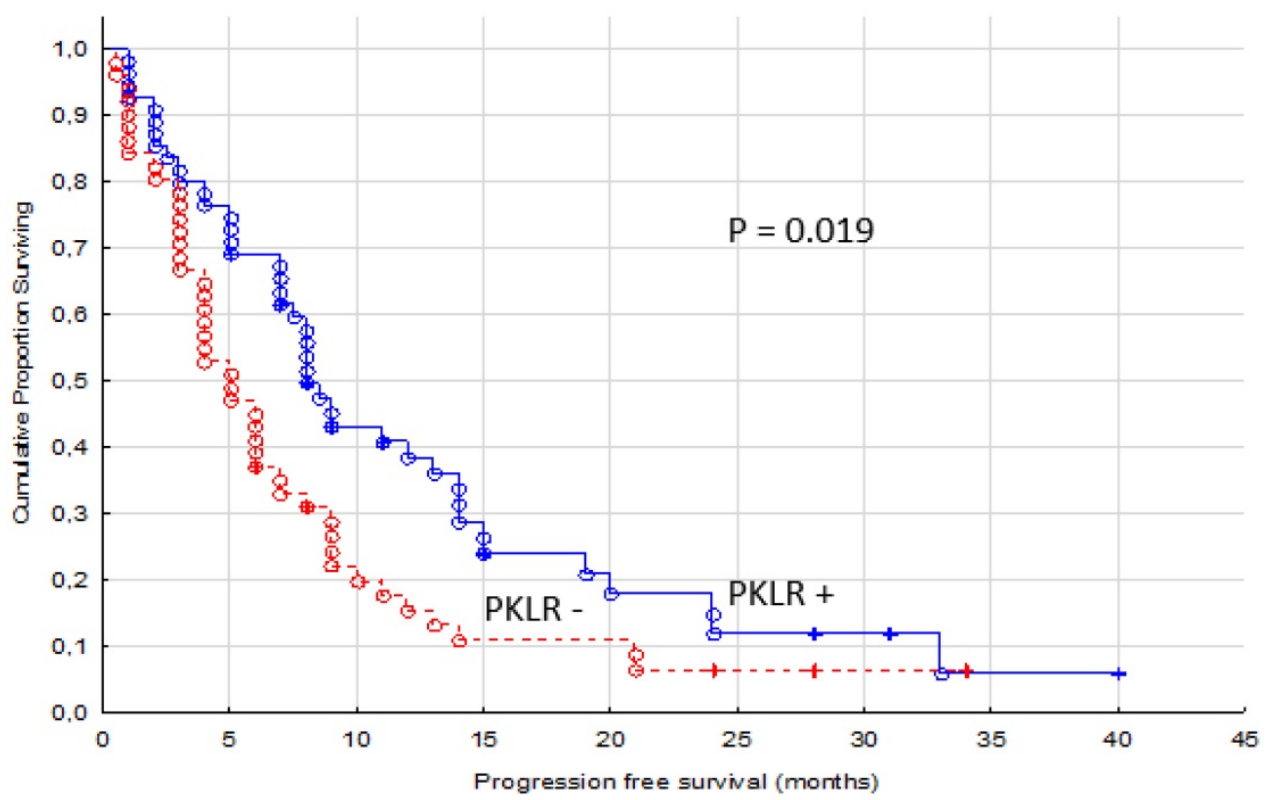

Figure 2. Progression free survival for renal cancer patients treated for metastatic disease with sunitinib or sorafenib in the first or second line setting, comparing membranous negative tumors (PKLR -, $n=51$ ) versus positive tumors (PKLR,$+ n=55)$ 
biomarker to predict the outcome of TKI-therapy is urgent. The aim of the present study was to investigate the potential value of tumoral expression of PKLR as a predictive marker for TKI treatment in mRCC patients.

In the majority of clear cell RCCs, the tumor suppressor gene von Hippel-Lindau (VHL) is mutated (31). As a result of this, hypoxia-inducible factor 1a (HIF-a) is accumulated causing VEGF and
PDGF overproduction (31). By binding these growth factors to and activating their receptors on the surface of vessels, vascular permeability and endothelial cell migration is stimulated. By blocking VEGF- and PDGF receptors with sunitinib and sorafenib, tumor angiogenesis is inhibited. Thereby growth and invasion is prevented counteracting metastatic spread $(32,33)$.

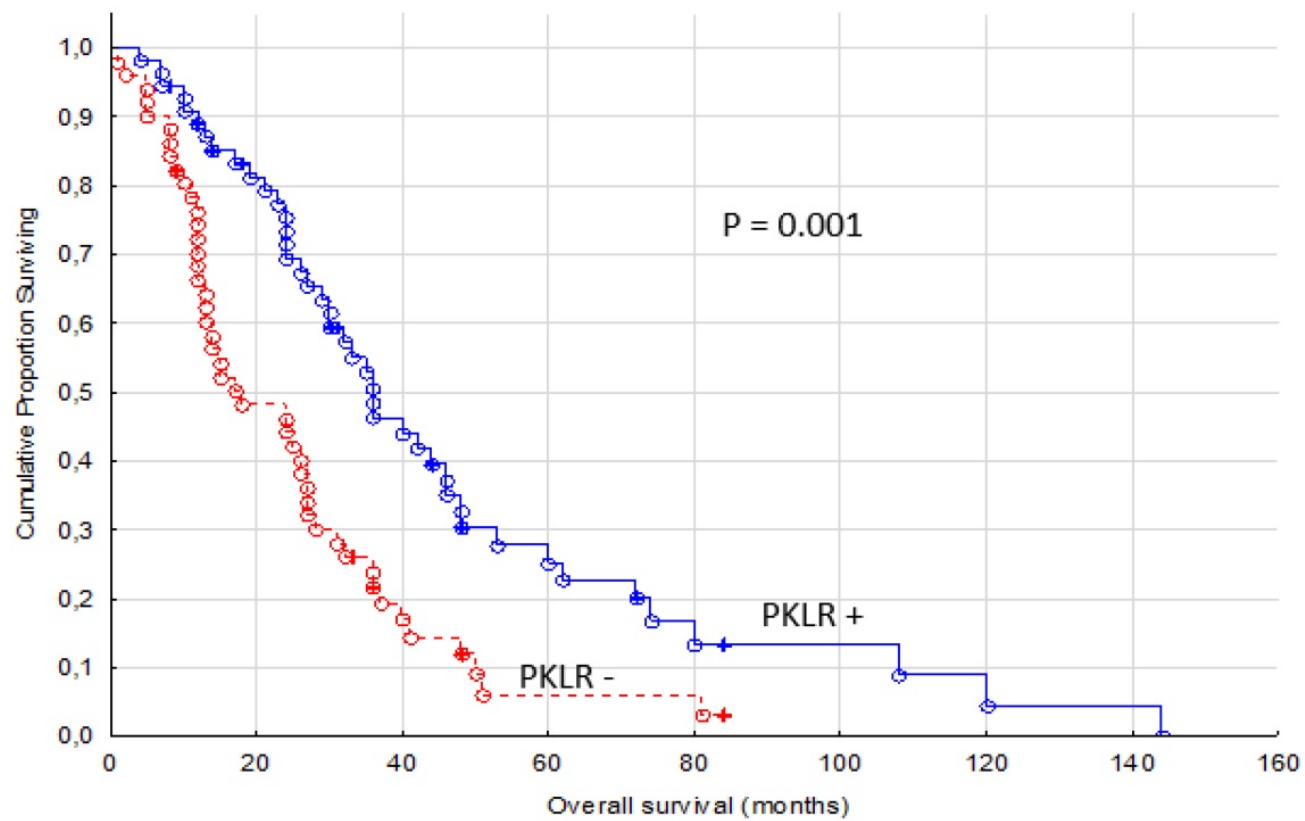

Figure 3. Overall survival for renal cancer patients treated for metastatic disease with sunitinib or sorafenib in the first or second line setting, comparing membranous negative tumors (PKLR -) $(\mathrm{n}=51)$ versus positive tumors (PKLR,$+ \mathrm{n}=55)$

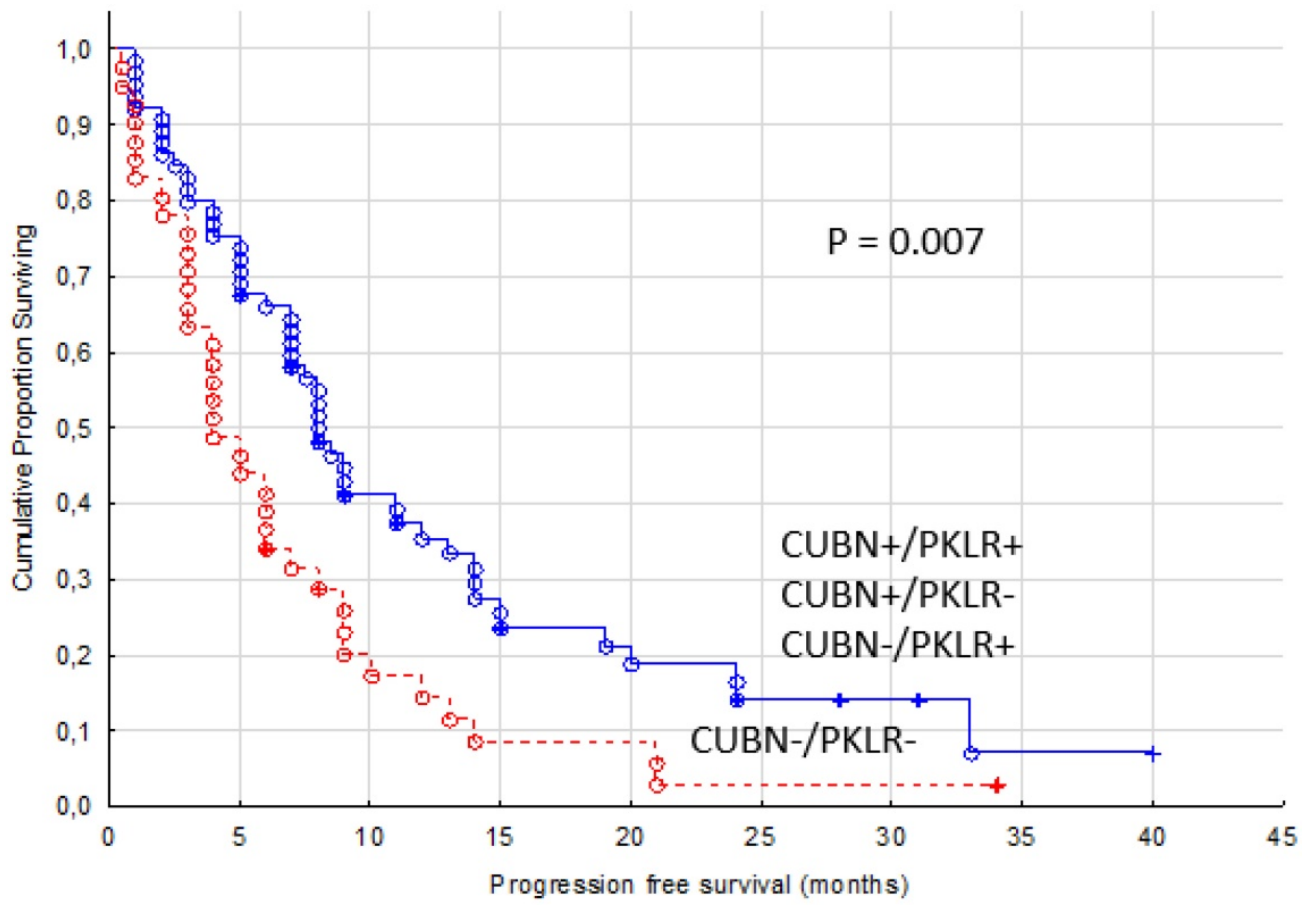

Figure 4. Progression free survival for renal cancer patients treated for metastatic disease with sunitinib or sorafenib in the first or second line setting, comparing membranous negative tumors (PKLR - and CUBN -, $n=41)$ versus other combinations of expression $(n=65)$ 
The number of studies evaluating predictors for TKI treatment is few and they have mainly concentrated on serum proteins. High baseline levels of VEGF and NGAL predicted a poor response to sunitinib therapy in mRCC patients $(n=85)$ (11). In contrast, patients with higher pre-treatment levels of VEGF $(n=177)$ benefitted significantly more from sorafenib therapy in terms of PFS than those with low levels $(n=535)(12)$.

Studying the tumor tissue is, in contrast with assessments of putative predictive markers in blood, a direct way to investigate potential target structures essential for treatment response. Only a few TMA-based studies for TKI prediction have been published and most of them with fewer patients than in the current study. Forty-two primary tumors from mRCC patients were investigated for expression of HIF-1a, CA-9, CD31, VEGFR1 and -2, pPDGFRa and $-\beta$ and Ki67 and the correlation to sunitinib response. Progression free survival was significantly longer in patients with high tumoral HIF-1a expression and VEGFR3 vessel staining (34). In contrast, OS was longer in patients with low PDGFRa score. A predictive value for tumor cell expression of programmed death-1 ligand (PD-L1) has also been demonstrated (35). Our earlier studies in the first- and second-line setting have indicated that CUBN is a predictor for both sunitinib and sorafenib therapy and cytoplasmic annexin A1 (ANXA1) for sunitinib-treatment $(26,27)$. We have also demonstrated that the combination of these two markers better than either marker alone can define patients with no treatment benefit at all (27).

In the HPA-project, PKLR was identified as a potential clinical biomarker based on its specific expression in both normal kidney and in RCC. Tumoral pyruvate kinase $L / R$ is important for glycolysis but little is known about its role in cancer (18). A prognostic value of PKM2, an isoenzyme to PKLR, in RCC patients has been demonstrated (23). In the present study we used a well-validated antibody to analyse the potential role of PKLR expression as a predictive marker for sunitinib and sorafenib medication in mRCC patients.

Our results indicate that membranous expression of PKLR in the primary tumors of mRCC patients acts as a marker of sunitinib and sorafenib benefit. In our study, tumoral PKLR expression resulted in almost doubled PFS compared with patients with no expression of PKLR. We also noticed a significantly longer OS in patients with PKLR positive tumors. Patients in these two groups (PKLR negative and PKLR positive tumors) may have been treated with different therapeutic agents after progression on TKI treatment. In addition, PKLR expression may also have a prognostic value. These two factors make it unclear whether the predictive value of PKLR extends to OS.

When analysing sunitinib and sorafenib treated patients separately, the statistical significance could only be recalled in the sunitinib treated group $(p=$ 0.01 ). This can be due to a lower number of patients in the sorafenib cohort $(n=45)$ but can also indicate that PKLR is a poorer predictor, or does not have any predictive value, for sorafenib treatment. The potential predictive value of PKLR for the other TKIs remains to be investigated.

In our earlier CUBN-study, patients treated with sunitinib $\leq 3$ months, defined as non-responders, had a significantly higher fraction of CUBN negative primary tumors (26). Therefore, we combined the expression of CUBN and PKLR and found that patients with no expression of these two markers were treated with sunitinib or sorafenib for four months in median (range 0.5-34). In comparison, patients with expression of either CUBN or PKLR or both experienced a doubled PFS (median eight months, range 1-40). Hence, our results indicate that the evaluation of the membranous expression of these two proteins together could even better than either alone help us to define the minor group of non-responders.

There are some limitations to this study. First, due to the retrospective study design, established prognostic markers were missing for many patients. Thus, the possible prognostic role of PKLR is impossible to analyse in our paper. However, the aim of the current study was not to find yet another prognostic marker, instead to evaluate the predictive role of PKLR. In this purpose, PFS was selected as the primary endpoint of the study. Second, since we only had access to the primary tumors, the potential heterogeneity of PKLR expression between primary tumor and metastases is unclear.

\section{Conclusions}

In summary, we show that PKLR tumoral expression is of predictive value for the treatment of mRCC patients since we found a significant association to PFS. In addition, combining our earlier findings on the CUBN protein, we demonstrate that these two markers together more accurately than either alone can define the non-responding group. Our results from this real world study need to be confirmed in larger studies before having impact on the clinical practise.

\section{Abbreviations}

ANXA1: Annexin A1; CUBN: Cubilin; HIF: Hypoxia-inducible factor; IF- a: Interferon alfa; 
MMP-9: Metalloproteinase-9; mRCC: Metastatic RCC; mTOR: Mammalian target of rapamycin; NGAL: Neutropfilgelatinase-associated lipocalin; OS: Overall survival; PDGF: Platelet-derived growth factor; PD-L1: Programmed death-1 ligand; PFS: Progression free survival; PKLR: pyruvate kinase liver and red blood cells; RCC: Renal cell carcinoma; SciLifeLab: Swedish Science for Life Laboratory; TKI: Tyrosine kinase inhibitor; TMA: Tissue microarray; TNFa: Tumor necrosis factor a; VEGF: Vascular endothelial growth factor; VHL: Von Hippel-Lindau.

\section{Acknowledgements}

We are grateful to all patients. The Research Foundation Stiftelsen Onkologiska Klinikens i Uppsala Forskningsfond has kindly supported this study. The study was also supported by grants from Lion's Cancer Fund and Knut and Alice Wallenberg Foundation.

\section{Competing Interests}

The authors have declared that no competing interest exists.

\section{References}

1. Motzer RJ, Bander NH, Nanus DM. Renal-cell carcinoma. N Engl J Med. 1996;335: 865-75

2. Negrier S, Escudier B, Lasset C, Douillard JY, Savary I, Chevreau C, et al. Recombinant human interleukin-2, recombinant human interferon alfa-2a, or both in metastatic renal-cell carcinoma. Groupe Francais d'Immunotherapie. N Engl J Med. 1998;338: 1272-8.

3. Motzer RJ, Hutson TE, et al. Sunitinib versus Interferon Alfa in Metastatic Renal-Cell Carcinoma. The New England Journal of Medicine. 2007; 115-24.

4. Escudier B, Porta C, Schmidinger M, Rioux-Leclercq N, Bex A, Khoo V, et al. Renal cell carcinoma: ESMO Clinical Practice Guidelines for diagnosis, treatment and follow-up. Ann Oncol. 2016;27: v58-v68.

5. Escudier B, Eisen T, Stadler WM, Szczylik C, Oudard S, Siebels M, et al. Sorafenib in advanced clear-cell renal-cell carcinoma. N Engl J Med. 2007;356: 125-34.

6. Di Lorenzo G, Porta C, Bellmunt J, Sternberg C, Kirkali Z, Staehler M, et al. Toxicities of targeted therapy and their management in kidney cancer. Eur Urol. 2011:59: 526-40.

7. Heng DY, Xie W, Regan MM, Warren MA, Golshayan AR, Sahi C, et al. Prognostic factors for overall survival in patients with metastatic renal cell carcinoma treated with vascular endothelial growth factor-targeted agents: results from a large, multicenter study. J Clin Oncol. 2009;27: 5794-9.

8. Szmit S, Zaborowska M, Wasko-Grabowska A, Zolnierek J, Nurzynski P, Filipiak KJ, et al. Cardiovascular comorbidities for prediction of progression free survival in patients with metastatic renal cell carcinoma treated with sorafenib. Kidney Blood Press Res. 2012;35: 468-76.

9. Nakano K, Komatsu K, Kubo T, Natsui S, Nukui A, Kurokawa S, et al. Hand foot skin reaction is associated with the clinical outcome in patients with metastatic renal cell carcinoma treated with sorafenib. Jpn J Clin Oncol. 2013;43: 1023-9.

10. Perez-Gracia JL, Prior C Guillen-Grima F Segura V, Gonzalez A, Panizo A, et al. Identification of TNF-alpha and MMP-9 as potential baseline predictive serum markers of sunitinib activity in patients with renal cell carcinoma using a human cytokine array. Br J Cancer. 2009;101: 1876-83.

11. Porta C, Paglino C, De Amici M, Quaglini S, Sacchi L, Imarisio I, et al Predictive value of baseline serum vascular endothelial growth factor and neutrophil gelatinase-associated lipocalin in advanced kidney cancer patients receiving sunitinib. Kidney Int. 2010;77: 809-15.

12. Escudier B, Eisen T, Stadler WM, Szczylik C, Oudard S, Staehler M, et al. Sorafenib for treatment of renal cell carcinoma: Final efficacy and safety results of the phase III treatment approaches in renal cancer global evaluation trial. J Clin Oncol. 2009;27: 3312-8.

13. Feng G, Ye X, Fang F, Pu C, Huang H, Li G. Quantification of plasma cell-free DNA in predicting therapeutic efficacy of sorafenib on metastatic clear cell renal cell carcinoma. Dis Markers. 2013;34: 105-11.

14. Noguchi T. [Regulation of pyruvate kinase gene expression and its clinical application]. Rinsho Byori. 1990;38: 868-75.
15. Yamada K, Tanaka T, Noguchi T. Members of the nuclear factor 1 family and hepatocyte nuclear factor 4 bind to overlapping sequences of the L-II element on the rat pyruvate kinase $\mathrm{L}$ gene promoter and regulate its expression. Biochem J. 1997;324: 917-25.

16. Israelsen WJ, Vander Heiden MG. Pyruvate kinase: Function, regulation and role in cancer. Semin Cell Dev Biol. 2015;43: 43-51.

17. Prakasam G, Singh RK, Iqbal MA, Saini SK, Tiku AB, Bamezai RNK. Pyruvate kinase $M$ knockdown-induced signaling via AMP-activated protein kinase promotes mitochondrial biogenesis, autophagy, and cancer cell survival. J Biol Chem. 2017;292: 15561-76.

18. Muirhead H. Isoenzymes of pyruvate kinase. Biochem Soc Trans. 1990;18: $193-6$.

19. Nie H, Li J, Yang XM, Cao QZ, Feng MX, Xue F, et al. Mineralocorticoid receptor suppresses cancer progression and the Warburg effect by modulating the miR-338-3p-PKLR axis in hepatocellular carcinoma. Hepatology. 2015;62: 1145-59.

20. Lesmana H, Dyer L, Li X, Denton J, Griffiths J, Chonat S, et al. Alu element insertion in PKLR gene as a novel cause of pyruvate kinase deficiency in Middle Eastern patients. Hum Mutat. 2018;39: 389-93.

21. Nguyen A, Loo JM, Mital R, Weinberg EM, Man FY, Zeng Z, et al. PKLR promotes colorectal cancer liver colonization through induction of glutathione synthesis. J Clin Invest. 2016;126: 681-94.

22. Baumunk D, Reichelt U, Hildebrandt J, Krause H, Ebbing J, Cash H, et al. Expression parameters of the metabolic pathway genes pyruvate dehydrogenase kinase-1 (PDK-1) and DJ-1/PARK7 in renal cell carcinoma (RCC). World J Urol. 2013;31: 1191-6.

23. Nisman B, Yutkin V, Nechushtan H, Gofrit ON, Peretz T, Gronowitz S, et al. Circulating tumor M2 pyruvate kinase and thymidine kinase 1 are potential predictors for disease recurrence in renal cell carcinoma after nephrectomy. Urology. 2010;76: 513.e1-6.

24. Ponten F, Schwenk JM, Asplund A, Edqvist PH. The Human Protein Atlas as a proteomic resource for biomarker discovery. J Intern Med. 2011;270: 428-46.

25. Gremel G, Djureinovic D, Niinivirta M, Laird A, Ljungqvist O, Johannesson H, et al. A systematic search strategy identifies cubilin as independent prognostic marker for renal cell carcinoma. BMC Cancer. 2017;17: 1-9.

26. Niinivirta M, Enblad G, Edqvist PH, Ponten F, Dragomir A, Ullenhag GJ. Tumoral cubilin is a predictive marker for treatment of renal cancer patients with sunitinib and sorafenib. J Cancer Res Clin Oncol. 2017;143: 961-70.

27. Niinivirta M, Enblad G, Edqvist PH, Ponten F, Dragomir A, Ullenhag GJ. Tumoral ANXA1 Is a Predictive Marker for Sunitinib Treatment of Renal Cancer Patients. J Cancer. 2017;8: 3975-83.

28. Kampf C, Olsson I, Ryberg U, Sjostedt E, Ponten F. Production of tissue microarrays, immunohistochemistry staining and digitalization within the human protein atlas. J Vis Exp. 2012

29. Ratain MJ, Eisen T, Stadler WM, Flaherty KT, Kaye SB, Rosner GL, et al. Phase II placebo-controlled randomized discontinuation trial of sorafenib in patients with metastatic renal cell carcinoma. J Clin Oncol. 2006;24: 2505-12.

30. Hutson TE, Bellmunt J, Porta C, Szczylik C, Staehler M, Nadel A, et al. Long-term safety of sorafenib in advanced renal cell carcinoma: follow-up of patients from phase III TARGET. Eur J Cancer. 2010;46: 2432-40.

31. van der Veldt AA, Boven E, Helgason HH, van Wouwe M, Berkhof J, de Gast G, et al. Predictive factors for severe toxicity of sunitinib in unselected patients with advanced renal cell cancer. Br J Cancer. 2008;99: 259-65.

32. Bergers G, Song S, Meyer-Morse N, Bergsland E, Hanahan D. Benefits of targeting both pericytes and endothelial cells in the tumor vasculature with kinase inhibitors. J Clin Invest. 2003;111: 1287-95.

33. Escudier B, Szczylik C, Porta C, Gore M. Treatment selection in metastatic renal cell carcinoma: expert consensus. Nat Rev Clin Oncol. 2012;9: 327-37.

34. Dornbusch J, Zacharis A, Meinhardt M, Erdmann K, Wolff I, Froehner M, et al. Analyses of potential predictive markers and survival data for a response to sunitinib in patients with metastatic renal cell carcinoma. PLoS One. 2013;8: e76386.

35. Choueiri TK, Figueroa DJ, Fay AP, Signoretti S, Liu Y, Gagnon R, et al. Correlation of PD-L1 tumor expression and treatment outcomes in patients with renal cell carcinoma receiving sunitinib or pazopanib: results from COMPARZ, a randomized controlled trial. Clin Cancer Res. 2015;21: 1071-7. 